\title{
MIT STANDARDS ZUM VIRTUELLEN FAHRZEUG
}

Wie können Embedded Systeme für unterschiedliche Fahrzeugplattformen wiederverwendet werden?

Wie lassen sich die Hürden zwischen den verschiedenen Funktionsdomänen reduzieren und mechatronische

Systeme in einer frühen Entwicklungsphase validieren?

Über die letzten zwei Jahrzehnte haben Automobilhersteller unermüdlich daran gearbeitet, ihre Produktentwicklungsprozesse zu optimieren.

Im Kontext des mechanischen Designs ist die virtuelle Modellierung kompletter Fahrzeuge inzwischen „gang und gäbe“. Mit Hilfe von 3D-Designanwendungen wie CATIA von Dassault Systèmes werden geometrische Bauteile virtuell in 3D erstellt und als vollständige Fahrzeuge in 3D Digital Mockups (DMUs) simuliert. Automobilhersteller wollen nun diesen Ansatz erweitern, um die Integration elektrischer und elektronischer Systeme einschließlich der Systemarchitektur und Embedded Software mit einzubeziehen. Damit soll sowohl die Steuerlogik von Embedded Systemen als auch deren Interaktion mit der Geometrie des Fahrzeugs sowie mit dessen Umgebung simuliert werden. Ein solches ,intelligentes' Digital Mockup wird als ,Functional Mockup' bezeichnet. Die Herausforderung liegt hier in der Wiederverwendung von Embedded Systemen unter Berücksichtigung der verschiedenen Fahrzeugmodelle, Produktreihen und Marken.

\section{AUTOSAR - BASIS FÜR DIE ENTWICKLUNG VON PRODUKTLINIEN}

Die Wiederverwendung von Embedded Systemen setzt die Nutzung des AUTOSAR-Standards voraus. Dieser bietet eine Implementierungsplattform, welche die Target-unabhängige Entwicklung von Steuergeräte-Software erlaubt. AUTOSAR Builder ${ }^{\odot}$ von Dassault Systèmes bietet hierfür einen offenen, flexiblen und leistungsstarken Anwendungs-Baukasten, der die schnelle und effiziente Modellierung, Validierung und Simulation von Embedded Systemen für definierte Steuerungssysteme (Target-ECU-s Electronic Control Units) ermöglicht.

\section{VIRTUELLE FAHRZEUGSIMULATION}

AUTOSAR Builder bietet u.a. eine Simulationsplattform, die sowohl White-Box als auch Blackbox-Testing der einzelnen Software Komponente sowie der vollständigen Software-Architektur unterstützt. Um das gesamte System oder auf Fahrzeug-Ebene zu skalieren muss man in der Lage sein, untergeordnete Systeme in einer einzigen Simulationsumgebung interoperabel zusammenstellen zu können. Diese Systeme können aus unterschiedlichsten Anwendungen eingebunden werden, einschließlich des Modelicabasierten Simulationswerkzeug Dymola (Dassault Systèmes), ASCET (ETAS) oder MATLAB/Simulink (The MathWorks) und auf Basis des FMI-Standards virtuell integriert werden.

Das Konzept der offenen FMI-Schnittstelle (Functional Mockup Interface) stammt aus dem Modelisar-Projekt, Teil der europäischen ITEA2-Initiative, mit dem erklärten Ziel, Designverfahren für Systeme und Embedded Software in Fahrzeugen zu verbes-

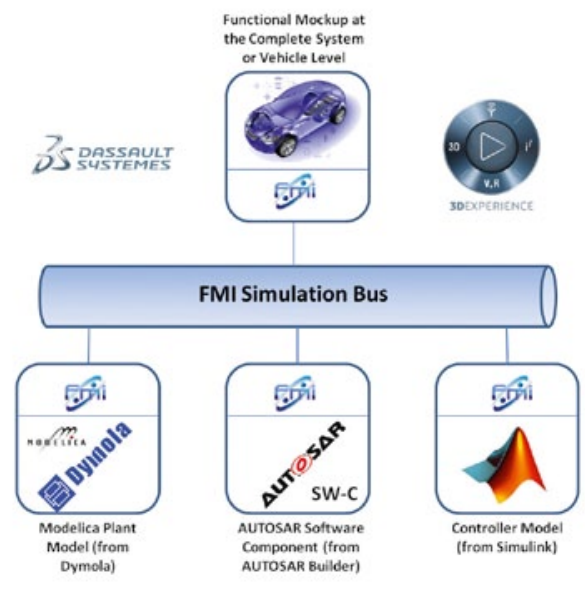

sern. FMI ist ein offener und herstellerunabhängiger SimulationsSchnittstellenstandard. Dieser erlaubt die virtuelle Integration von Submodellen, die aus unterschiedlichen Modellierungswerkzeugen exportiert wurden. Da sowohl AUTOSAR Builder als auch DYMOLA den FMI-Standard unterstützen, wird so die Validierung komplexer Systeme auf Basis von Simulation ermöglicht.

\section{ARCHITEKTUR FÜR EINEN GANZHEITLICHEN ANSATZ ZUR WIEDERVERWENDUNG}

Eine solche Lösung verlangt einen integrierten Ansatz für die Entwicklung, Simulation und Validierung integrierter elektrischer, softwarebasierter und mechanischer Fahrzeugsysteme. Dieser kann durch das Design, De-Komposition und Wiederverwendung komplexer Embedded Systeme auf der Grundlage eines mehrschichtigen Ansatzes verwirklicht werden. Dieser Ansatz umfasst folgende Bereiche:

- Kundenbezogene \& technische Funktionen

- Funktions- \& Softwarenetze

- Hardwaretopologie

- Software-Partitionierung (Definition der Topologie und der Schnittstellen)

- Simulation der Embedded System-Architektur Die 3DEXPERIENCE-Plattform von Dassault Systèmes bietet Entwicklungsteams von Fahrzeug-Plattformen eine Integrations- und Simulationsumgebung für die bereichsübergreifende Zusammenarbeit. Sie nutzt die leistungsstarken Anwendungswerkzeuge AUTOSAR Builder, Dymola, CATIA, FMI \& Reqtify (Requirement Tracing) auf einer einheitlichen Plattform mit ausgereiften Funktionen für die Verwaltung von Daten, Lifecycles, Konfigurationen, Zugriffsrechten, Governance, sowie für die Projekt- und Programm-Steuerung.

Für weitere Informationen, besuchen Sie bitte: 3ds.com/AUTOSAR-Builder 